\title{
Altered brain language network in idiopathic peripheral facial paralysis patients with dysarthria
}

\author{
Wenwen Gao ${ }^{1 \#}$, Xiaowei Han ${ }^{1 \#}$, Haimei Li ${ }^{2 \#}$, Yijiang Zhu ${ }^{3}$, Lei Du ${ }^{1}$, Yuli Wang ${ }^{1}$, Sumin Shi ${ }^{1}$, Jing Liu ${ }^{1}$, \\ Chao $\mathrm{Fu}^{1}$, Lu Zhang ${ }^{4}$, Guolin $\mathrm{Ma}^{1}$ \\ ${ }^{1}$ Department of Radiology, China-Japan Friendship Hospital, Beijing, China; ${ }^{2}$ Department of Radiology, Fu Xing Hospital, Capital Medical \\ University, Beijing, China; ${ }^{3}$ Department of Imaging, Anhui Provincial Hospital, Hefei, China; ${ }^{4}$ Department of Science and Education, Shangluo \\ Central Hospital, Shangluo, China \\ Contributions: (I) Conception and design: G Ma; (II) Administrative support: G Ma; (III) Provision of study materials or patients: Y Zhu, W Gao, H \\ Li, L Du; (IV) Collection and assembly of data: X Han, Y Wang, S Shi, J Liu, C Fu; (V) Data analysis and interpretation: W Gao, X Han, H Li; (VI) \\ Manuscript writing: All authors; (VII) Final approval of manuscript: All authors. \\ \#These authors contributed equally to the study. \\ Correspondence to: Guolin Ma. Department of Radiology, China-Japan Friendship Hospital, No.2 East Yinghua Road, Chaoyang District, Beijing, \\ China. Email: maguolin1007@qq.com.
}

Background: Dysarthria is one of the common symptoms of facial paralysis (FP). This study aimed to investigate functional alterations in the brain language network in early idiopathic peripheral FP patients with dysarthria using resting-state functional magnetic resonance imaging (fMRI).

Methods: A total of 45 cases of FP (left 22, right 23) and 34 cases of healthy control (HC) were recruited into this study. The data of patients with left-side FP and matched controls (17 cases) were flipped from left to right, and the brain regions were defined as ipsilateral and contralateral regions. The FC of 16 ROIs in classical language centers and regions that may be involved in language function were calculated. After identifying the differences of FC between the two groups, the correlation analysis between altered FC and TFGS score of oral muscle movement in FP group were analyzed.

Results: The FC between bilateral language regions has a significantly decreased trend in FP group compared with HC group $(\mathrm{P}<0.05)$. The ipsilateral inferior frontal gyrus, superior temporal gyrus, and middle temporal gyrus exhibited significantly decreased FC with multiple brain regions. In addition, we found that thalamus and cerebellum also with a significant alteration in FC in FP patients indicating that these two regions may also be involved in the mechanism of dysarthria in FP. The correlation analysis results indicated that the decrease of FC was positively correlated with the severity of oral paralysis.

Conclusions: Idiopathic peripheral FP with dysarthria induces several FC alterations in the brain language network. The severity of oral paralysis is associated with these functional alterations.

Keywords: Dysarthria; facial paralysis (FP); functional connectivity; language; resting-state functional magnetic resonance imaging (fMRI)

Submitted Aug 30, 2019. Accepted for publication Feb 27, 2020.

doi: $10.21037 /$ atm.2020.03.133

View this article at: http://dx.doi.org/10.21037/atm.2020.03.133

\section{Introduction}

Idiopathic peripheral facial paralysis (FP) is a disease of facial muscle dysfunction caused by facial nerve injury (1). Its clinical manifestations are mainly concentrated in face, which can lead to a serious psychological impact on patients (2). Dysarthria is one of the common symptoms of idiopathic peripheral FP, but less attention has been paid to the neural mechanism in language alterations in the patients. Early identification in functional changes of the brain language network in FP patients with dysarthria 
will be of great significance to understand the pathogenesis of the disease and provide early diagnosis and timely treatment.

Language expression is achieved through a high degree of neuromuscular coordination in vocal organs. The pronunciation process is that the motor cortex emits a pulse through the cortical brainstem bundle into the brainstem nucleus, and then transmits through the cerebral nerve to the vocal organs to make it move and produce a sound. Besides, the vocal organs also accept regulated impulse from cerebellum and basal ganglia (3), any interruption along the pathway will induce language dysfunction. Language dysfunction includes aphasia and dysarthria. Brain tissue damage such as cerebrovascular disease (4) and brain tumor (5) can lead to aphasia, while dysarthria is related to vocal muscle dysfunction.

Resting-state fMRI, as a non-invasive method, has been used to observe neuron activity by identifying changes in the local oxygen consumption and the cerebral blood flow in brain regions. Functional connectivity (FC) is an fMRIbased index characterized the inter-regional synchronization of intrinsic brain activity, which has been wildly used to investigate the difference of brain activity between clinical and healthy population (6). By using resting-state fMRI, researchers found $\mathrm{FC}$ changes in several diseases with language dysfunction. van der Salm et al. found alterations in FC of the language network in patients with aphasia after a stroke (7). Children with autism spectrum disorder (ASD) combined language impairment also have been reported as atypical FC of the language network (8). Structural abnormalities in language regions were found in patients with clefts of the lip and/or palate with dysarthria (9). Many studies have shown that there may be more brain regions involved in language function than in the classical language center. Recent studies have shown that the basal ganglia $(10,11)$, cerebellum (8), thalamus (12), and pons (13) are all involved in language function.

For assessing functional alterations in the brain language network in early idiopathic peripheral FP patients with dysarthria, we calculated FC of the language network in patients and controls. Classical language centers and regions that may be involved in language function previously mentioned were analyzed. Additionally, to explore correlations between altered brain language network and severity of oral paralysis in patients, we evaluated the facial function in participants using Toronto Facial Grading System (TFGS) (14) and analyzed the correlations between altered FC and TFGS score of oral muscle movement in FP group.

\section{Methods}

\section{Subjects}

Forty-five patients with FP and 34 healthy controls (HC) participated in the study. This study was approved by the Ethics Committee of our hospital, and all subjects have given informed consent before the experiment. The patients with FP came from our hospital from 2017 to 2018. The inclusion criteria were: adult unilateral idiopathic peripheral FP patients with dysarthria diagnosed by clinicians; onset time within 7 days; no other craniocerebral lesions and psychiatric diseases; being right-handed. The exclusion criteria were: central FP; patients with other vocal muscle disorders such as muscle weakness, hemifacial spasm. There were 22 cases of left FP and 23 cases of right FP in patient group, 17 males and 28 females, with an average age of $43 \pm 13$ years (22-66 years). The HC group were recruited from society and inclusion criteria were: no language dysfunction assessed by clinicians; no neurological and psychiatric illness, and family history; being right-handed. There were 34 cases in control group, 14 males and 20 females, with an average age of $46 \pm 14$ years (24-68 years). The TFGS scores were used to evaluate the facial function for all subjects by clinicians (Table 1).

\section{MRI data acquisition}

A 3.0 T MRI scanner (GE, Discovery MR750, Milwaukee, United States) with a standard head coil was used to acquire the data. All subjects underwent a T2WI scan to exclude craniocerebral organic diseases. Before fMRI scanning, each subject was told to close their eyes, rest and avoid thinking during the exam. The resting-state fMRI with a single-shot gradient recalled echo-planar imaging sequence: slice thickness $=3.5 \mathrm{~mm}$, slice spacing $=0.7 \mathrm{~mm}$, repetition time $(\mathrm{TR})=2,000 \mathrm{~ms}$, echo time $(\mathrm{TE})=30 \mathrm{~ms}$, flip angle $=90$ degrees, matrix $=64 \times 64$, field of view $(\mathrm{FOV}$ ) $=224 \mathrm{~mm} \times 224 \mathrm{~mm}$, number of excitations $(\mathrm{NEX})=1,34$ slices and 240 phases. 3D T1WI anatomic images were reconstructed using three-dimensional fast spoiled gradientecho sequences (3D FSPGR): slice thickness $=1.0 \mathrm{~mm}$, TR $=6.7 \mathrm{~ms}, \mathrm{TE}=\min$ full, matrix $=256 \times 256, \mathrm{FOV}=256 \mathrm{~mm} \times$ $256 \mathrm{~mm}, \mathrm{NEX}=1$.

\section{Data pre-processing}

Before any pre-processing steps, we flipped the data of patients with left-side FP and the matched controls 
Table 1 Demographic and clinical data of participants

\begin{tabular}{lccc}
\hline Variables & FP group $(n=45)$ & HC group $(n=34)$ & $P$ value \\
\hline Age (years) & $43.20 \pm 13.39$ & $46.18 \pm 14.38$ & $0.346^{\dagger}$ \\
Sex (male/female) & $17 / 28$ & $14 / 20$ & $0.759^{\ddagger}$ \\
Education (years) & $12.80 \pm 4.41$ & $14.03 \pm 4.38$ & $0.222^{\dagger}$ \\
Duration (days) & $4.40 \pm 1.79$ & 0 & - \\
TFGS (scores) & $16.89 \pm 16.04$ & $100.00 \pm 0.00$ & - \\
\hline
\end{tabular}

${ }^{\dagger}$, independent two-sample $t$-test; ${ }^{\ddagger}$, Chi-square test. The lower the TFGS scores, the more severe the symptoms of facial paralysis. FP, facial paralysis; HC, healthy control; TFGS, Toronto Facial Grading System.

(17 cases) along the Y-axis, in order to directly compare them with the right-side FP patients $(15,16)$. The restingstate fMRI data were pre-processed in Data Processing Assistant for Resting-State fMRI (DPARSF) software (17). Briefly, the pre-processing steps were as follows: the DICOM data was converted to NIFTI format; the first 10 time points were removed; after slice timing correction and realignment, the functional images were normalized by using T1 images unified segmentation to the Montreal Neurological Institute (MNI) standard brain; the cases with head motion exceeds $2 \mathrm{~mm}$ or $2^{\circ}$ were excluded; the $4 \mathrm{~mm}$ Gaussian kernel was used for spatial smoothing; and the low-frequency drift and high-frequency noise were removed by band-pass filtering $(0.01-0.08 \mathrm{~Hz})$. After pre-processing, the individual data were used for further analysis.

\section{Definition of region of interest (ROI)}

To explore functional changes in language network in idiopathic peripheral FP patients with dysarthria, we included classical language centers and regions that may be involved in language function (caudate, cerebellum, thalamus, and pons) as ROIs. The basal ganglia group and the language group in Stanford University Willard 499 fROI atlas (18) were used because these two groups covered the above brain regions. These two atlas groups contain a total of 16 ROIs and the voxel size of each ROI is $3 \mathrm{~mm} \times 3 \mathrm{~mm}$ $\times 3 \mathrm{~mm}$ (Table 2, Figure 1).

\section{Network connectivity analysis}

Before calculating FC, six head motion parameters obtained by rigid body correction, signals of white matter and cerebrospinal fluid were removed as covariables by linear regression. The FC was calculated based on the Pearson correlation of all voxels BOLD signals for each ROI using
Rest toolbox (http://resting-fmri.sourceforge.net/). Fisher's r-to-z transformation was utilized to increase the normality of data distribution and each subject obtained a $16 \times 16 \mathrm{FC}$ (z values) matrix (Figure 2). For each z value, Two-sample T-test was conducted on SPSS 20.0 software (SPSS Inc., Chicago, IL, USA) between the two groups. Age, gender, and education were regressed as covariates. False discovery rate (FDR) correction was used to control false positives for multiple comparisons and $\mathrm{P}<0.05$ was considered as statistical significance (Figure 3). Spearman correlations between altered FC and TFGS score of oral muscle movement (open mouth smile) in patients were analyzed.

\section{Results}

\section{Clinical results}

Sex, age, and education level were not significantly different between FP group and HC group $(\mathrm{P}>0.05)$ (Table 1).

\section{Functional connectivity analysis}

Ipsilateral inferior frontal gyrus exhibited decreased FC with multiple regions in patients. The ipsilateral inferior frontal triangularis (IFGtriang) had decreased FC with bilateral inferior frontal orbitalis (ORBinf), contralateral superior temporal gyrus (STG), angular gyrus (ANG), and ipsilateral STG, while it had increased FC with ipsilateral middle temporal gyrus (MTG) in FP group compared with HC group. The FC between bilateral ORBinf was also decreased in patients (Figure 4A).

Thalamus and cerebellum had an alteration in FC in FP group. The FC between ipsilateral thalamus and contralateral IFGtriang was increased, while the FC between contralateral thalamus and contralateral supramarginal gyrus (SMG) was decreased in FP group. 
Table 2 Information of 16 ROIs

\begin{tabular}{|c|c|c|c|c|c|}
\hline Willard fROI 499 atlas & Brain regions & Cluster size & \multicolumn{3}{|c|}{ MNI coordinates } \\
\hline \multirow[t]{6}{*}{ Basal Ganglia } & CAU.I & 175 & 14 & 10 & 14 \\
\hline & THA.I & 200 & 10 & -12 & 10 \\
\hline & THA.C & 257 & -10 & -12 & 10 \\
\hline & IFGtriang.C & 18 & -44 & 22 & 24 \\
\hline & IFGtriang.I & 63 & 49 & 30 & 18 \\
\hline & Pons & 32 & -5 & -25 & -38 \\
\hline \multirow[t]{7}{*}{ Language } & ORBinf.C & 323 & -48 & 29 & -7 \\
\hline & SMG.C & 201 & -55 & -48 & 31 \\
\hline & ANG.C & 340 & -55 & -59 & 28 \\
\hline & ORBinf.I & 58 & 51 & 28 & -8 \\
\hline & STG.I & 281 & 56 & -45 & 19 \\
\hline & MTG.I & 569 & 51 & -22 & -8 \\
\hline & CERE.crus1.C & 99 & -22 & -76 & -31 \\
\hline
\end{tabular}

".I" means the ipsilateral to the facial paralysis, ".C" means the contralateral to the facial paralysis. CAU, caudate; THA, thalamus; IFGtriang, inferior frontal triangularis; ORBinf, inferior frontal orbitalis; MTG, middle temporal gyrus; STG, superior temporal gyrus; SMG, supramarginal gyrus; ANG, angular gyrus; CERE.crus1, cerebellar crus 1 area.

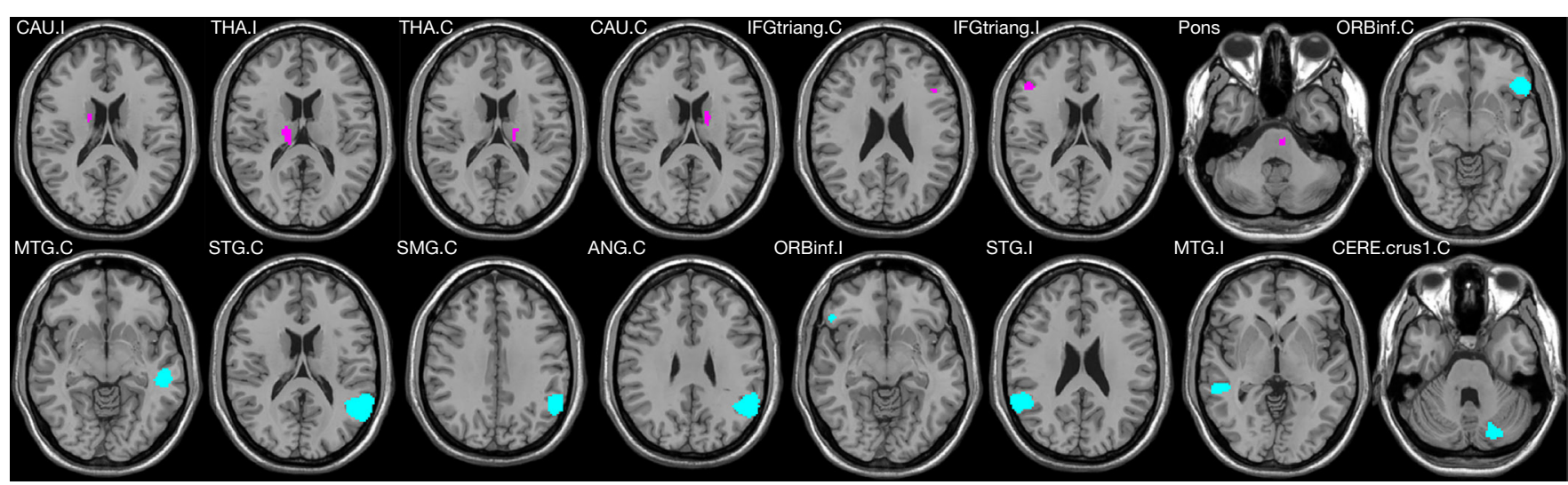

Figure 1 The ROI illustration of the basal ganglia group and the language group in the Stanford University Willard 499 fROI atlas.

The contralateral cerebellar crus 1 area had increased FC with ipsilateral STG and MTG in patients (Figure 4B).

Ipsilateral temporal gyrus showed decreased FC with multiple regions in patients. The ipsilateral STG had decreased FC with contralateral STG, SMG, and ANG, and the ipsilateral MTG had decreased FC with contralateral STG and ANG in FP group compared with HC group (Figure 4C). 
A

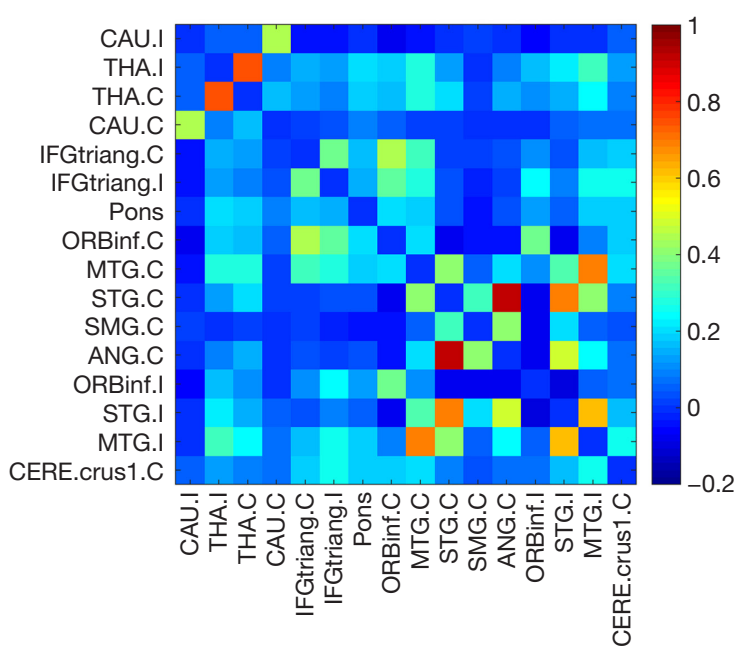

C

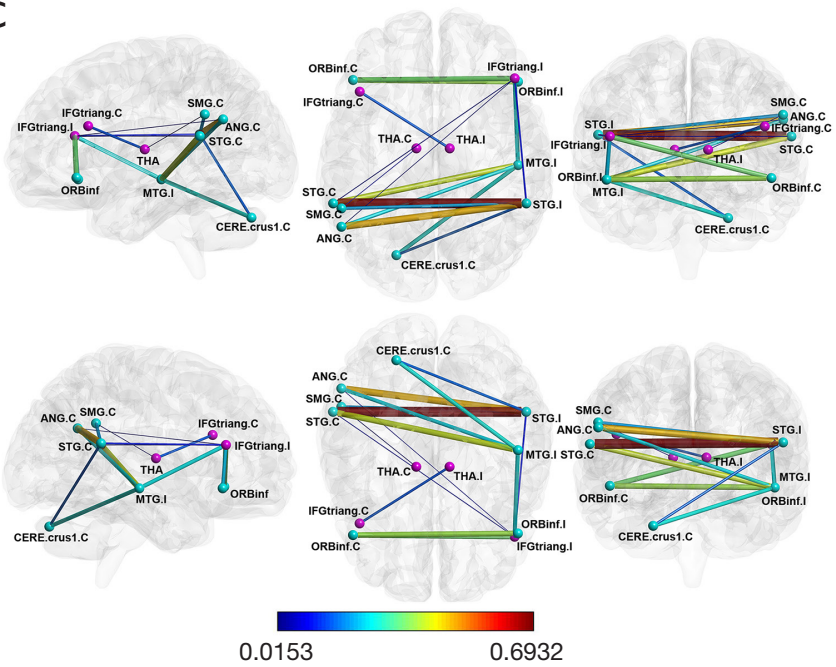

B

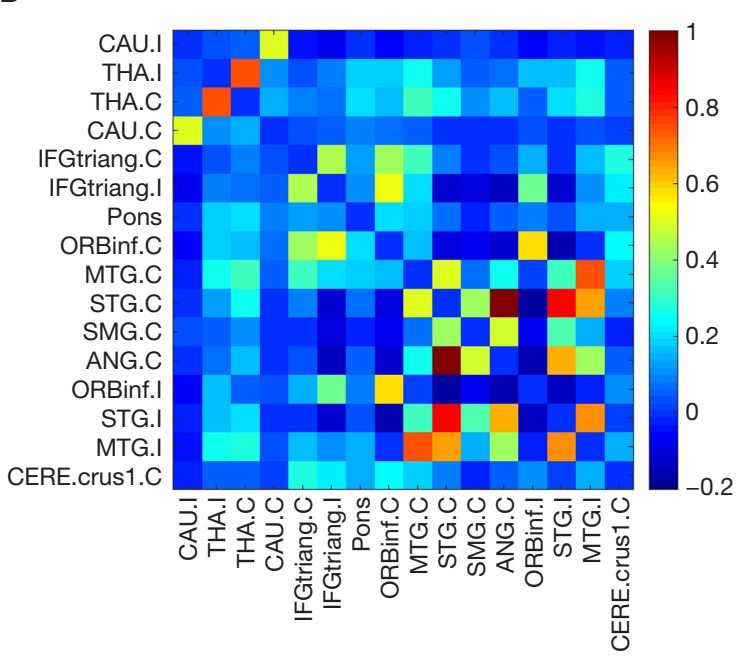

D

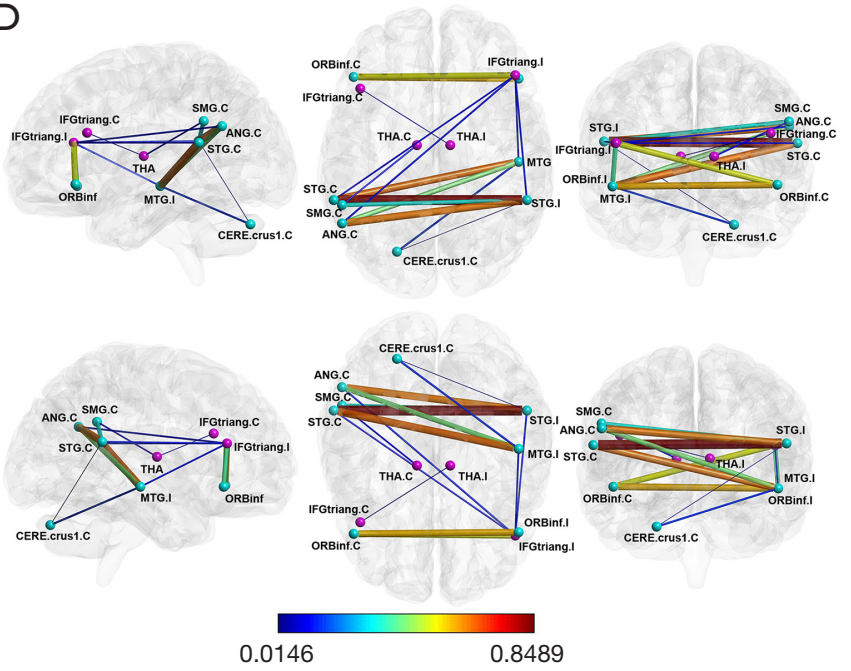

Figure 2 The FC was calculated based on the Pearson correlation for each ROI and Fisher's r-to-z transformation was conducted. (A,B) The FC matrix in FP group and HC group, color grids represent mean $\mathrm{z}$ values. (C,D) The brain regions and FC with significant differences between the two groups, purple nodes represent the basal ganglia group, green nodes represent language group, color and thickness of edges represent absolute $\mathrm{z}$ values.

\section{Correlations between altered FC and TFGS score of oral muscle movement}

The FC between bilateral STG correlated positively with TFGS score of oral muscle movement $(\mathrm{r}=0.514, \mathrm{P}=0.000)$ (Figure 5A). Additionally, FC between ipsilateral STG and contralateral ANG showed positive correlation with the TFGS score $(\mathrm{r}=0.468, \mathrm{P}=0.001)$ (Figure $5 B)$, and the correlation result of FC between ipsilateral MTG and contralateral STG with TFGS score was positive similarly $(\mathrm{r}=0.302, \mathrm{P}=0.044)$ (Figure 5C).

\section{Discussion}

\section{Altered FC of language network in FP group}

The present study investigated the functional alterations in the brain language network in early idiopathic peripheral FP patients with dysarthria. We found that FC between bilateral language regions has a significantly decreased trend in FP group compared with HC group $(\mathrm{P}<0.05)$. The ipsilateral inferior frontal gyrus (IFG), STG, and MTG had decreased FC with multiple brain regions. Besides, thalamus and cerebellum also with a significant alteration in $\mathrm{FC}$ in 
A

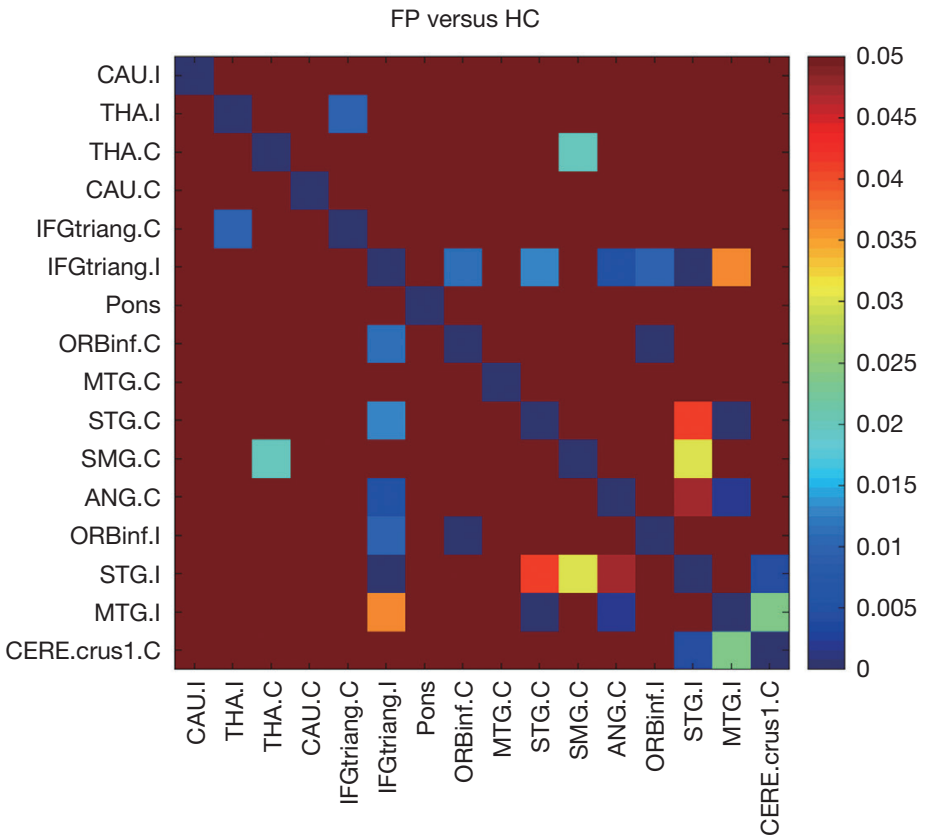

B
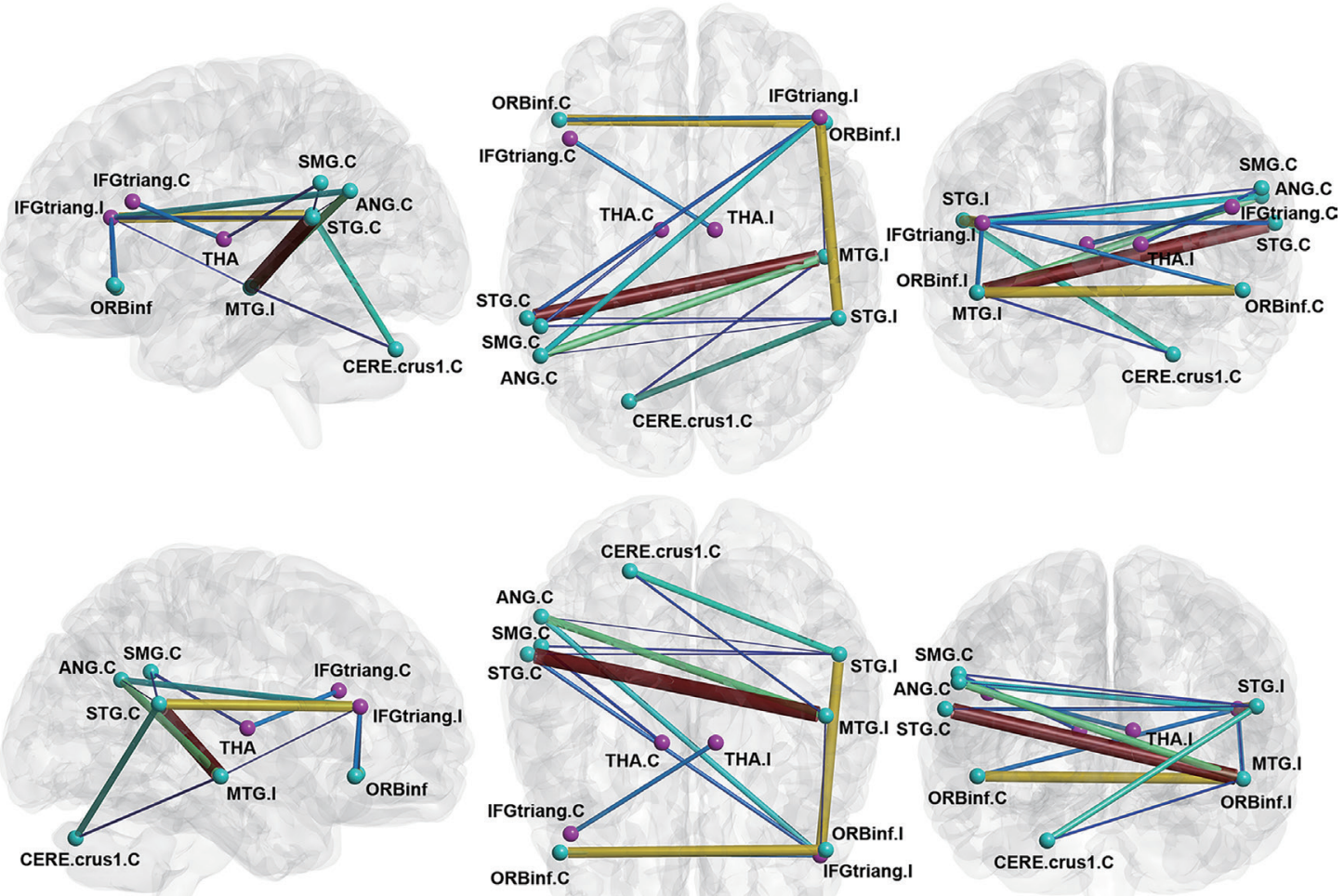

2.014

4.53

Figure 3 Two-sample T-test was conducted to compare the differences in FC between the two groups and the threshold was set at $\mathrm{P}<0.05$ FDR (false discovery rate) corrected. (A) The color grid represents the P value of each FC. (B) The brain regions and FC with significant differences between the two groups, purple nodes represent the basal ganglia group, green nodes represent the language group, color and thickness of edges represent $t$ values. 
A

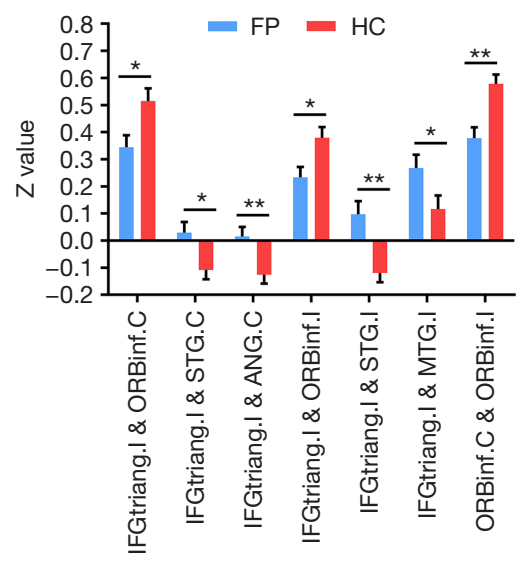

B

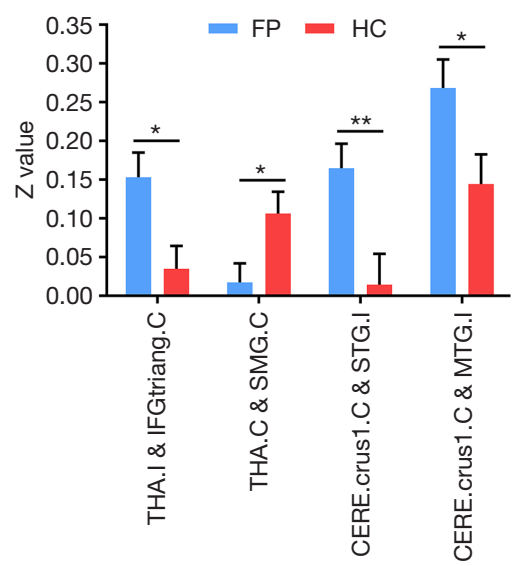

C

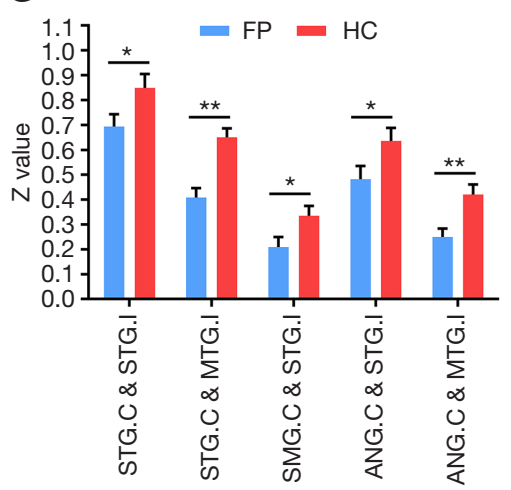

Figure 4 The FC of the brain regions had a significant difference between the two groups (FDR corrected at $\mathrm{P}<0.05$ ). Error bars depict standard error of the mean. * $\mathrm{P}<0.05$; ${ }^{*}, \mathrm{P}<0.01$. Stars indicate a significant difference. (A) The ipsilateral IFGtriang had decreased FC with multiple brain regions and the FC between bilateral ORBinf was also decreased in FP group; (B) the thalamus and the cerebellar crus1 area had altered FC with other brain regions in FP group; (C) the FC between Wernicke area and contralateral brain regions was decreased in FP group.

A

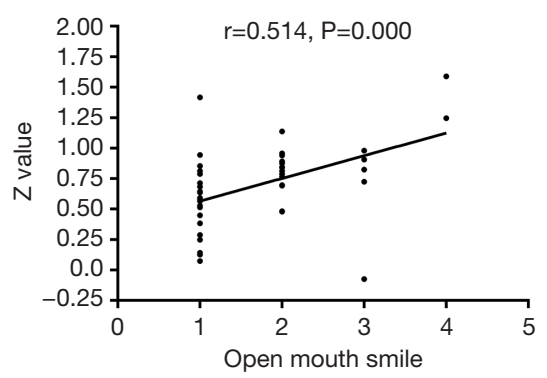

B

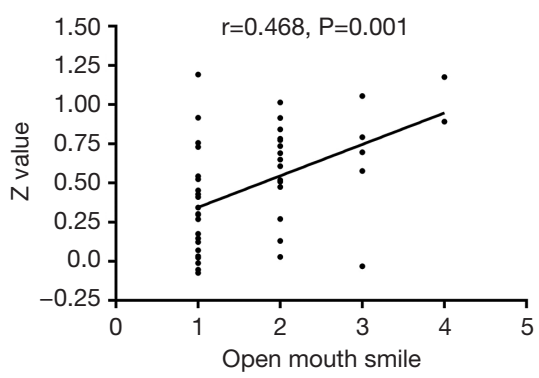

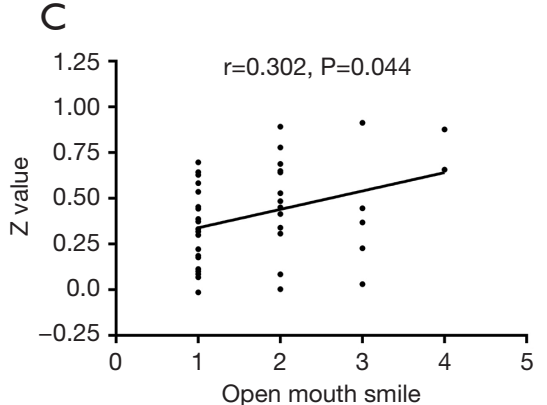

Figure 5 The Spearman correlation analysis results indicated that altered FC was correlated with TFGS score of oral muscle movement (open mouth smile) in FP group. The number 1 to 4 of the "open mouth smile" indicates the ipsilateral oral muscle movement compared with the normal side and the lower the score, the more severe the degree of oral paralysis: 1: no movement; 2: mild movement; 3: moderate movement; 4: almost complete movement. (A) The FC between bilateral STG showed a positive correlation with TFGS score of open mouth smile $(\mathrm{r}=0.514, \mathrm{P}=0.000)$. (B) The FC between ipsilateral STG and contralateral ANG showed a positive correlation with TFGS score of open mouth smile $(\mathrm{r}=0.468, \mathrm{P}=0.001)$. (C) The FC between ipsilateral MTG and contralateral STG correlated positively with TFGS score of open mouth smile $(\mathrm{r}=0.302, \mathrm{P}=0.044)$.

FP patients.

Our results show that ipsilateral IFGtriang had significant decreased FC with multiple brain regions in FP group, and FC between bilateral ORBinf was also decreased in patients. Left IFGtriang and ORBinf were found activated in word retrieval (19) and the syntactic and semantic procession of written language (20), while right ORBinf plays a positive role in the regulation of the language expression (21).
We speculate that the dysfunction of language expression caused by FP induced the decrease of functional activity in IFGtriang and ORBinf. Although traditionally thinking right-handed people have a left dominance of language $(22,23)$, the right hemisphere provides a better basis for language recovery when multiple cerebral damaging (24), suggesting that both hemispheres are involved in language recovery (25). Additionally, aphasia occurs in left cerebral 
infarction supporting the theory of left dominance of language in right-handed people (26-28), while right cerebral infarction can also lead to aphasia indicating that bilateral hemispheres are involved in language function (29). FC changes of IFGtriang and ORBinf in patients indicating that these two regions are involved in language expression and there is a bilateral language dominance, which is in accordance with previous studies.

In our results, $\mathrm{FC}$ between ipsilateral thalamus and contralateral IFGtriang was increased, and FC between contralateral thalamus and contralateral SMG was decreased in FP group. As we know, the thalamus is a center of speech processing between Broca's area and Wernicke's area, its injury can lead to changes in cognition and speech. Aphasia can occur in unilateral thalamic injury, indicating that thalamus plays an important role in the process of language generation (12). Our results show that bilateral thalamus with an alteration in FC suggesting the procession of speech in thalamus also is bilateral. The decrease of functional activity in ipsilateral IFGtriang may lead to increased activity in contralateral IFGtriang as compensation, thus induced the increase of $\mathrm{FC}$ between contralateral IFGtriang and ipsilateral thalamus. The decrease of $\mathrm{FC}$ between contralateral thalamus and contralateral SMG may be due to the decrease of functional activity caused by dysarthria associated with FP.

In addition, we also found ipsilateral STG had decreased FC with contralateral STG, SMG, and ANG, and ipsilateral MTG had decreased FC with contralateral STG and ANG, while contralateral cerebellar crus 1 area had increased FC with ipsilateral STG and MTG in FP group. STG, MTG, SMG, and ANG all belong to the Wernicke area (30). Left SMG and STG are involved in speech processing and the posterior part of left STG and ANG are related to semantic processing, and the middle part of left MTG and anterior part of STG are involved in sentence processing (31). Our results show that FC between Wernicke area and contralateral brain regions is reduced, which is consistent with previous studies emphasizing both hemispheres are involved in language generation and understanding $(32,33)$. The dysfunction of language expression in patients with dysarthria may lead to a decrease of functional activity in these brain regions, thus induce the decrease of FC. Cerebellum also was found involved in language function $(34,35)$ and its infarction (36) and injury (37) can lead to dysarthria. In FP group, functional activity of STG and MTG were decreased, and cerebellum may increase the functional activity as compensation.

\section{Relationship between altered FC and severity of oral paralysis}

In this study, we also investigated correlations between the altered brain language network and the severity of oral paralysis in patients. The results show that the decrease of FC in the Wernicke area (STG, MTG, ANG) was correlated with the severity of oral paralysis, suggesting that dysarthria caused by facial nerve paralysis may lead to the decrease of neural activity in the brain language network. Early identification in these alterations would give a piece of evidence for possible language rehabilitation training in patients.

\section{Limitations}

There were several limitations in our study. First, this study was limited to small sample size and patients with left FP and right FP were all included. To control the problem on different side of the lesion, we flipped the left FP group from left to right. Functional alterations in the brain language network in left FP or right FP can be analyzed separately in the future. Second, we used a cross-section study design to explore the alterations in language network, future longitudinal studies would help us to understand the mechanism of these changes in patients with $\mathrm{FP}$ at different disease stages. Finally, we used resting-state data and without design language tasks to observe the changes in language network in patients in real-time. Task-state fMRI can be used to continue the study in the future.

\section{Conclusions}

In conclusion, our results show that FC between bilateral language regions has a significantly decreased trend in FP group compared with HC group $(\mathrm{P}<0.05)$, and the severity of oral paralysis was correlated positively with the decrease of FC. This study provides a piece of evidence that dysarthria caused by facial nerve paralysis may lead to a decrease of neural activity in the brain language network. Early identification in these changes would give a basis for understanding the pathogenesis of the disease and providing early diagnosis and possible treatment.

\section{Acknowledgments}

Funding: This work was supported by the National Natural Science Foundation of China [No. 81971585, 81571641] and The National Key Research and Development Program 
of China (No. 2016YFC1307001, 2019YFC0120903).

\section{Footnote}

Provenance and Peer Review: This article was commissioned by the Guest Editors (Haotian Lin and Limin Yu) for the series "Medical Artificial Intelligent Research" published in Annals of Translational Medicine. The article was sent for external peer review organized by the Guest Editors and the editorial office.

Conflicts of Interest: All authors have completed the ICMJE uniform disclosure form (available at http://dx.doi. org/10.21037/atm.2020.03.133). The series "Medical Artificial Intelligent Research" was commissioned by the editorial office without any funding or sponsorship. The authors have no other conflicts of interest to declare.

Ethical Statement: The authors are accountable for all aspects of the work in ensuring that questions related to the accuracy or integrity of any part of the work are appropriately investigated and resolved. This study was approved by the Ethics Committee of China-Japan Friendship Hospital, and all subjects have given informed consent before the study.

Open Access Statement: This is an Open Access article distributed in accordance with the Creative Commons Attribution-NonCommercial-NoDerivs 4.0 International License (CC BY-NC-ND 4.0), which permits the noncommercial replication and distribution of the article with the strict proviso that no changes or edits are made and the original work is properly cited (including links to both the formal publication through the relevant DOI and the license). See: https://creativecommons.org/licenses/by-nc-nd/4.0/.

\section{References}

1. Basić-Kes V, Dobrota VD, Cesarik M, et al. Peripheral facial weakness (Bell's palsy). Acta Clin Croat 2013;52:195-202.

2. Han X, Li H, Wang X, et al. Altered Brain Fraction Amplitude of Low Frequency Fluctuation at Resting State in Patients With Early Left and Right Bell's Palsy: Do They Have Differences? Front Neurosci 2018;12:797.

3. Ullman MT. A neurocognitive perspective on language: the declarative/procedural model. Nat Rev Neurosci 2001;2:717-26.
4. Tao J, Fang $\mathrm{Y}, \mathrm{Wu} Z$, et al. Community-applied research of a traditional Chinese medicine rehabilitation scheme on Broca's aphasia after stroke: study protocol for a randomized controlled trial. Trials 2014;15:290.

5. Recht LD, Mccarthy K, O"Donnell BF, et al. Tumorassociated aphasia in left hemisphere primary brain tumors: The importance of age and tumor grade. Neurology 1989;39:48-50.

6. Yan CG, Yang Z, Colcombe SJ, et al. Concordance Among Indices of Intrinsic Brain Function: Insights from InterIndividual Variation and Temporal Dynamics. Science Bulletin 2017;62:1572-84.

7. van der Salm SM, van der Meer JN, Nederveen AJ, et al. Functional MRI study of response inhibition in myoclonus dystonia. Exp Neurol 2013;247:623-9.

8. Verly M, Verhoeven J, Zink I, et al. Altered functional connectivity of the language network in ASD: Role of classical language areas and cerebellum. Neuroimage Clin 2014;4:374-82.

9. Nopoulos P, Berg S, Canady J, et al. Structural brain abnormalities in adult males with clefts of the lip and/or palate. Genet Med 2002;4:1-9.

10. Grönholm EO, Roll MC, Horne MA, et al. Predominance of caudate nucleus lesions in acute ischaemic stroke patients with impairment in language and speech. Eur J Neurol 2016;23:148-53.

11. Kotz SA, Schmidt-Kassow M. Basal ganglia contribution to rule expectancy and temporal predictability in speech. Cortex 2015;68:48-60.

12. De Witte L, Brouns R, Kavadias D, et al. Cognitive, affective and behavioural disturbances following vascular thalamic lesions: A review. Cortex 2011;47:273-319.

13. Urban PP, Stoeter P. 1.6. Dysarthria due to ischemic brainstem lesions. Clin Neurophysiol 2007;118:2807.

14. Kayhan FT, Zurakowski D, Rauch SD. Toronto Facial Grading System: Interobserver reliability. Otolaryngol Head Neck Surg 2000;122:212-5.

15. Song W, Cao Z, Lang C, et al. Disrupted functional connectivity of striatal sub-regions in Bell's palsy patients. Neuroimage Clin 2017;14:122-9.

16. Klingner CM, Volk GF, Brodoehl S, et al. The effects of deefferentation without deafferentation on functional connectivity in patients with facial palsy. Neuroimage Clin 2014;6:26-31.

17. Yan CG, Wang XD, Zuo XN, et al. DPABI: Data Processing \& Analysis for (Resting-State) Brain Imaging. Neuroinformatics 2016;14:339-51.

18. Richiardi J, Altmann A, Milazzo AC, et al. BRAIN 
NETWORKS. Correlated gene expression supports synchronous activity in brain networks. Science 2015;348:1241-4.

19. Jeon HA, Lee KM, Kim YB, et al. Neural substrates of semantic relationships: Common and distinct leftfrontal activities for generation of synonyms vs. antonyms. Neuroimage 2009;48:449-57.

20. Schafer RJ, Constable T. Modulation of functional connectivity with the syntactic and semantic demands of a Noun Phrase Formation Task: A possible role for the Default Network. Neuroimage 2009;46:882-90.

21. Van Ettinger-Veenstra H, Ragnehed M, Mcallister A, et al. Right-hemispheric cortical contributions to language ability in healthy adults. Brain Lang 2012;120:395-400.

22. De Witte L, Hecke WV, Dua G, et al. LP50: Atypical cerebral language dominance in a right-handed patient: an anatomoclinical study with DES, fMRI and DTI. Clin Neurol Neurosurg 2014;117:12-21.

23. Jacques P, Alban L, Barbu Stéphanie, et al. Challenges Facing the Study of the Evolutionary Origins of Human Right-Handedness and Language. Int J Primatol 2018;39:183-207.

24. Crosson B, Mcgregor K, Gopinath KS, et al. Functional MRI of Language in Aphasia: A Review of the Literature and the Methodological Challenges. Neuropsychol Rev 2007;17:157-77.

25. Skipper-Kallal LM, Lacey EH, Shihui X, et al. Right Hemisphere Remapping of Naming Functions Depends on Lesion Size and Location in Poststroke Aphasia. Neural Plast 2017;2017:8740353.

26. Whitwell JL, Duffy JR, Strand EA, et al. Distinct regional anatomic and functional correlates of neurodegenerative apraxia of speech and aphasia: An MRI and FDG-PET study. Brain Lang 2013;125:245-52.

27. Yourganov G, Smith KG, Fridriksson J, et al. Predicting aphasia type from brain damage measured with structural

Cite this article as: Gao W, Han X, Li H, Zhu Y, Du L, Wang Y, Shi S, Liu J, Fu C, Zhang L, Ma G. Altered brain language network in idiopathic peripheral facial paralysis patients with dysarthria. Ann Transl Med 2020;8(11):699. doi: 10.21037/atm.2020.03.133
MRI. Cortex 2015;73:203-15.

28. Kiran S, Meier EL, Kapse KJ, et al. Changes in task-based effective connectivity in language networks following rehabilitation in post-stroke patients with aphasia. Front Hum Neurosci 2015;9:316.

29. Tan X, Guo Y, Dun S, et al. Crossed aphasia following cerebral infarction in a right-handed patient with atypical cerebral language dominance. J Neurol 2018;265:1671-5.

30. Salek KE, Hassan IS, Kotrotsou A, et al. Silent Sentence Completion Shows Superiority Localizing Wernicke's Area and Activation Patterns of Distinct Language Paradigms Correlate with Genomics: Prospective Study. Sci Rep 2017;7:12054.

31. Vigneau M, Beaucousin V, Hervé PY, et al. Meta-analyzing left hemisphere language areas: Phonology, semantics, and sentence processing. Neuroimage 2006;30:1414-32.

32. Silbert LJ, Honey CJ, Simony E, et al. Coupled neural systems underlie the production and comprehension of naturalistic narrative speech. Proc Natl Acad Sci U S A 2014;111:E4687-96.

33. Lambon Ralph MA, Pobric G, Jefferies E. Conceptual Knowledge Is Underpinned by the Temporal Pole Bilaterally: Convergent Evidence from rTMS. Cereb Cortex 2009;19:832-8.

34. E KH, Chen SH, Ho MH, et al. A meta-analysis of cerebellar contributions to higher cognition from PET and fMRI studies. Hum Brain Mapp 2014;35:593-615.

35. Peter Mariën, Beaton A. The enigmatic linguistic cerebellum: clinical relevance and unanswered questions on nonmotor speech and language deficits in cerebellar disorders. Cerebellum Ataxias 2014;1:12.

36. Ogawa K, Suzuki Y, Oishi M, et al. Two patients with isolated dysarthria caused by cerebellar infarction. Cent Eur J Med 2013;8:725-9.

37. Lechtenberg R, Gilman S. Speech disorders in cerebellar disease. Ann Neurol 1978;3:285-90. 